fixed point free involution on $S^{i}$ and the identity on the other component, it is easy to see that:

$$
H_{1}\left(\frac{S^{1} \times S^{2}}{T_{1}}, Z_{2}\right) \neq H_{1}\left(\frac{S_{1} \times S_{2}}{T_{2}}, Z_{2}\right) .
$$

\title{
BIBLIOGRAPHY
}

1. A. V. Černavskiř, The impossibility of a strictly continuous two-to-one partition of a homology cube, Dokl. Akad. Nauk SSSR 144 (1962), 286-289=Soviet Math. Dokl. 3 (1962), 726-729.

2. G. W. Henderson, Abstract 601-48, Notices Amer. Math. Soc. 10 (1963), 349.

3. P. Civin, Two to one maps of manifolds, Duke Math. J. 10 (1945), 49-57.

4. P. A. Smith, Transformations of finite period. I, Ann. of Math. 40 (1938), 127-164.

Rutgers, The State University

\section{VON NEUMANN REGULARITY IN JORDAN ALGEBRAS}

CHESTER E. TSAI

In a Jordan algebra $J$ (not necessarily finite-dimensional), an element $a$ is regular if there exists an element $x$ in $J$ such that $x U_{a}=2(a \cdot x) \cdot a-a^{2} \cdot x=a$. The algebra is a regular algebra if every element in $J$ is regular (see $\left[1\right.$, p. 246]). If $J=A^{+}$, where $A$ is an associative algebra, then $a$ is regular in $J$ if, and only if, it is von Neumann regular in the associative algebra $A$; that is if there exists an element $x$ in $A$ such that $a x a=a$. The purpose of this note is to carry out some analogous results for Jordan algebras (not necessarily special Jordan algebras). The characteristic of the ground field $K$ is always assumed not to be two.

Throughout this paper, the following three identities will be used quite frequently.

$$
\begin{aligned}
{[(x \cdot y) \cdot d] \cdot z+[(x \cdot z) \cdot d] \cdot y+[(y \cdot z) \cdot d] \cdot x=(x \cdot y) \cdot(d \cdot z) } & \\
& +(x \cdot z) \cdot(d \cdot y)+(y \cdot z) \cdot(d \cdot x) \text { for all } x, y, z, d \text { in } J . \\
U_{x U_{y}}= & U_{y} U_{x} U_{y} \quad \text { for all } x, y \text { in } J . \\
U_{x U_{a}-a}= & U_{a} U_{x} U_{a}+U_{a}-2 L(a \cdot x) U_{a}-2 L(a) L(x) U a \\
& +2 L(x) L(a) U a \quad \text { for all } a, x \text { in } J .
\end{aligned}
$$

Identity (1) is the linearized form of the Jordan identity $x^{2} \cdot(x \cdot y)$ $=x \cdot\left(x^{2} \cdot y\right)$. Identity (2), which is called the fundamental formula of Jordan algebras, was proved in several places, (see, for example, [2]).

Received by the editors July 11, 1966. 
For the proof of the identity (3), we shall first show

$$
U_{a, x U_{a}}=(L(x \cdot a)+L(a) L(x)-L(x) L(a)) L_{a} .
$$

If $J=A^{+}$is a special Jordan algebra, then for any $y$ in $J$ we have

$$
\begin{aligned}
2 y U_{a, x U_{a}=} & \left(x U_{a}\right) y a+a y\left(x U_{a}\right) \\
= & a x a y a+a y a x a \\
= & \frac{1}{2} a(x a y+a x y+y x a+y a x+y a x+a y x+x y a \\
& \quad \quad \quad+x a y-y x a-x y a-a x y-a y x) a \\
= & 2 a[(x \cdot a) \cdot y+(y \cdot a) \cdot x-(x \cdot y) \cdot a] a \\
= & 2 y(L(x \cdot a)+L(a) L(x)-L(x) L(a)) U_{a} .
\end{aligned}
$$

When $J$ is an arbitrary Jordan algebra, the identity (4) is also true. The validity is yielded by MacDonald's Theorem (see [2], [3]), which states that any identity which holds for all special Jordan algebras and which does not involve more than three variables and is of degree at most one in one of these variables is valid for all Jordan algebras.

Now we can show (3) by direct computation.

$$
\begin{aligned}
U_{x U_{a-a}}= & U_{x U_{a}}+U_{a}-2 U_{x U_{a}, a}=U_{a} U_{x} U_{a}+U_{a} \\
& -2(L(a \cdot x)+L(a) L(x)-L(x) L(a)) U_{a} .
\end{aligned}
$$

McCoy's Lemma. In a Jordan algebra $J$, if $x U_{a}-a$ is regular for some $x, a$ in $J$, then $a$ is regular.

PROOF. Let $x U_{a}-a=y U_{\left(x U_{a}-a\right)}$, then

$$
\begin{aligned}
a & =x U_{a}-y U_{\left(x U_{a}-a\right)} \\
& =\left[x-y U_{a} U_{x}+2 y L(a \cdot x)+2 y L(a) L(x)-2 y L(x) L(a)-y\right] U_{a} .
\end{aligned}
$$

Thus $a$ is regular.

Theorem 1. Let $J$ be a Jordan algebra and $D$ be an ideal in $J$. Then $J$ is regular if, and only if, both $D$ and $J / D$ are regular.

Proof. If $J$ is regular, $J / D$ is a homomorphic image of $J$, thus $J / D$ is regular. Furthermore, if $a$ is in $D$, there exists $d$ in $J$ such that $d U_{a}=a$. But then $a=d U_{a}=d U_{d U_{a}}=d U_{a} U_{d} U_{a}=y U_{a}$, where $y=d U_{a} U_{d}$ is in $D$. Since $D$ is an ideal, thus $a$ is regular (in $D$ ).

Conversely, suppose both $D$ and $J / D$ are regular, and let $a$ be an element in $J$. Let $\bar{a}=a+D$ and $\bar{x}=x+D$ such that $\bar{a}=\bar{x} U_{a}$. Then $a-x U_{a} \in D$, but $D$ is regular, hence, by McCoy's lemma, $a$ is regular. 
Corollary. Let $J$ be a Jordan algebra and $D_{1}, D_{2}$ be ideals in $J$ such that $J / D_{1}$ and $J / D_{2}$ are both regular. Then $J / D_{1} \cap D_{2}$ is regular.

Proof. Since $\left(D_{1}+D_{2}\right) / D_{2}$ is an ideal in $J / D_{2}$, its regularity is proved by the theorem. But $\left(D_{1}+D_{2}\right) / D_{2} \cong D_{1} / D_{1} \cap D_{2}$, the latter, thus, is also regular. Finally, applying the theorem to $J / D_{1}$ $\cong J /\left(D_{1} \cap D_{2}\right) / D_{1} /\left(D_{1} \cap D_{2}\right)$, we show $J / D_{1} \cap D_{2}$ is regular.

Lemma 2. The sum of a finite number of regular ideals in $J$ is a regular ideal in $J$.

Proof. One needs to show $D_{1}+D_{2}$ is regular if both $D_{1}$ and $D_{2}$ are. However, this assertion is clear by the isomorphism $D_{1}+D_{2} / D_{1}$ $\cong D_{2} / D_{1} \cap D_{2}$ and Theorem 1 .

Theorem 2. Any Jordan algebra $J$ has a unique maximal regular ideal $M . J / M$ has no nonzero regular ideal.

Proof. The set $M$ of elements of finite sums each lying in some regular ideal of $J$ is an ideal of $J$. Every element of $M$ is regular.

If $M^{\prime}$ is a regular ideal in $J$, by definition of $M, M^{\prime}+M \subseteq M$, so $M^{\prime} \subseteq M$. Thus $M$ is the unique maximal regular ideal in $J$.

If $J / M$ has a regular ideal $\bar{D}$, then $\bar{D}=D / M$ where $D$ is an ideal in $J$. Since $\bar{D}$ and $M$ are both regular so is $D$, by Theorem 1 . Hence $D \subseteq M$ and $D=M$ and $\bar{D}=0$.

The author is in debt to the referee for his invaluable suggestion.

\section{BiBLIOGRAPHY}

1. N. Jacobson, Structure theory for a class of Jordan algebras, Proc. Nat. Acad. Sci. U.S.A. 55 (1966), 243-251.

2. - A coordinatization theorem for Jordan algebras, Proc. Nat. Acad. Sci. U.S.A. 48 (1962), 1154-1160.

3. I. G. MacDonald, Jordan algebras with three generators, Proc. London Math. Soc. 10 (1960), 395-408.

Michigan State University 